\title{
Petri Nets, Discrete Physics, and Distributed Quantum Computation
}

\author{
Samson Abramsky \\ Oxford University Computing Laboratory
}

This paper is dedicated to Ugo Montanari on the occasion of his 65th birthday.

\begin{abstract}
We shall describe connections between Petri nets, quantum physics and category theory. The view of Net theory as a kind of discrete physics has been consistently emphasized by Carl-Adam Petri. The connections between Petri nets and monoidal categories were illuminated in pioneering work by Ugo Montanari and José Meseguer. Recent work by the author and Bob Coecke has shown how monoidal categories with certain additional structure (dagger compactness) can be used as the setting for an effective axiomatization of quantum mechanics, with striking applications to quantum information. This additional structure matches the extension of the Montanari-Meseguer approach by Marti-Oliet and Meseguer, motivated by linear logic.
\end{abstract}

\section{Introduction}

In this paper, we shall be concerned with links between three, prima facie very different, areas:

- Models of concurrent computation, especially Petri nets.

- Physics, especially quantum mechanics and quantum information.

- Monoidal categories with additional structure (e.g. compact closure [16]).

In particular, we are motivated by the following previous work:

- Petri's seminal work, which has always emphasized links between his Net Theory and Physics [24-26].

- The pioneering work by Ugo Montanari and José Meseguer [21] using monoidal categories as a setting for Net Theory, further extended by Marti-Oliet and Meseguer [20].

- Our own work with Bob Coecke [4,5], using monoidal categories as a setting for a novel axiomatization of quantum mechanics, with applications to quantum information.

Thus the situation can be depicted as follows: 


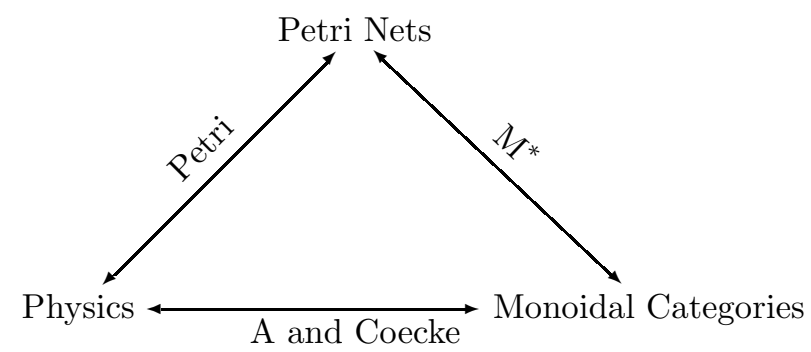

\section{Petri Nets as Discrete Physics}

An important quality of Petri's conception of concurrency, as compared with "linguistic" approaches such as process calculi, is that it seeks to explain fundamental concepts: causality, concurrency, process, etc. in a syntax-independent, "geometric" fashion. Another important point, which may originally have seemed merely eccentric, but now looks rather ahead of its time, is the extent to which Petri's thinking was explicitly influenced by physics (see e.g. [25]). As one example, note that K-density comes from one of Carnap's axiomatizations of relativity [11]. To a large extent, and by design, Net Theory can be seen as a kind of discrete physics: lines are time-like causal flows, cuts are space-like regions, process unfoldings of a marked net are like the solution trajectories of a differential equation.

This acquires new significance today, when the consequences of the idea that "Information is Physical" [17] are being explored in the rapidly developing field of quantum informatics. Moreover, the need to recognize the spatial structure of distributed systems has become apparent, and is made explicit in formalisms such as the Ambient calculus [10], and Milner's bigraphs [23].

We shall illustrate these points with some quotations from [25].

"This paper attempts to provide a common basis for physical and computational ways of thinking. ... If this approach should turn out to be a small, but definite step towards the remote (perhaps illusory) goal of founding technology and natural sciences on a theory of information flow, the author would feel rewarded beyond merit."

The paper discusses four levels of description for processes and systems. We concentrate on the first two.

\section{Level 0: Concurrency Structure}

"Concurrency is short for "the binary relation of cotemporality of world points". Here we follow closely the axiomatizations of relativistic space-time ...

For individuals, we take the time layers of signals, the smallest propagators of physical effects. Some signals are particles, others propagate 
...through interaction. The history of each signal is a "world line" and consists of world points. Let $x$ and $y$ be individuals; we write $x<y$ if $x \neq y$ and a signal passes from $x$ to $y$. We define

$$
\begin{aligned}
& x \operatorname{co} y \Leftrightarrow \text { neither } x<y \text { nor } y<x \\
& x \text { li } y \Leftrightarrow x<y \text { or } y<x \text { or } x=y
\end{aligned}
$$

Let

$$
\operatorname{Co}(x):=\{z \mid x \operatorname{co} z\} \quad \operatorname{Li}(x):=\{z \mid x \text { li } z\}
$$

If $\operatorname{Co}(x)=\operatorname{Co}(y)$ or $\operatorname{Li}(x)=\operatorname{Li}(y)$, we shall collect $x$ and $y$ into a cluster; such clusters are equivalence classes of world points, and will be the individuals of Level 1."

\section{Level 1: Occurrence Nets}

"We shall now describe the structure of the set $X$ of all occurrences, and its partition into a set $S$ of state elements, and a set $T$ of transition elements. ...

A subset $l \subset X$ will be called a Line iff it is a maximal set of occurrences which are pairwise in relation li.

A subset $c \subset X$ will be called a Cut iff it is a maximal set of occurrences which are pairwise in relation co.

The old physical postulate that every Cut represents a spatial distribution ... can now be written as 'every Cut meets every Line', i.e. as K-density."

\subsection{Causal Sets and Other Roads}

Quite independently, physicists have recently been thinking along strikingly similar lines, in one of the radical current approaches to quantum gravity, which is being developed by Raphael Sorkin and his collaborators [28, 9].

Following intuitions going back to Riemann and Einstein, the aim with Causal Sets is to build a theory of space-time which is ultimately (at the "Planck scale") discrete. A causal set is just a locally finite poset. The elements are events, the ordering is causality. The aim is to build everything back from these ingredients, under the slogan

$$
\text { Order }+ \text { Number }=\text { Geometry. }
$$

"Number" here refers to counting the events which have occurred in a given region of spacetime; this is meaningful by local finiteness, and leads to a notion of "volume". There is a "dynamics" which comes from the growth of a poset. Large-scale structural properties of space-time should emerge from stochastic properties of such growth.

One may also note the popular book by Lee Smolin [27]: the discussion in the first few chapters, especially of the relational view of spacetime, is very much in the same spirit. 
We also mention the striking recent work by Keye Martin and Prakash Panangaden [19], which builds back the spacetime manifold from the causal order, using ideas from domain theory.

\section{Interlude: Symmetric Monoidal Categories}

We briefly recall and motivate the basic setting of symmetric monoidal categories. For further details, we refer to standard texts such as [18].

\subsection{Categories}

A category $\mathcal{C}$ has objects (types) $A, B, C, \ldots$, and for each pair of objects $A$, $B$ a set of morphisms $\mathcal{C}(A, B)$. (Notation: $f: A \rightarrow B)$. It also has identities $\operatorname{id}_{A}: A \rightarrow A$, and composition $g \circ f$ when types match:

$$
A \stackrel{f}{\longrightarrow} B \stackrel{g}{\longrightarrow} C
$$

Categories allow us to deal explictly with typed processes, e.g.

\begin{tabular}{|c|c|c|}
\hline Logic & Programming & Computation \\
\hline Propositions & Data Types & States \\
\hline Proofs & Programs & Transitions \\
\hline
\end{tabular}

\subsection{Symmetric Monoidal Categories}

A symmetric monoidal category comes equipped with an associative operation $\otimes$, the "tensor product", which acts on both objects and morphisms - a bifunctor:

$$
A \otimes B \quad f_{1} \otimes f_{2}: A_{1} \otimes A_{2} \longrightarrow B_{1} \otimes B_{2}
$$

There is also a symmetry operation

$$
\sigma_{A, B}: A \otimes B \longrightarrow B \otimes A
$$

which satisfies some 'obvious' rules, e.g. naturality:

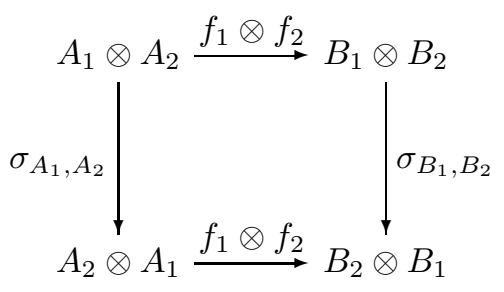


The Logic of Tensor Product Tensor can express independent or concurrent actions (mathematically: bifunctoriality):

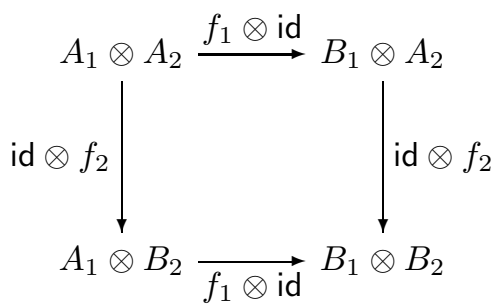

But tensor is not a 'cartesian' or categorical product, in the sense that we cannot reconstruct an 'element' of the tensor from its components.

This turns out to comprise the absence of natural diagonals (copying) and projections (deleting):

$$
\begin{array}{cc}
A \stackrel{\Delta}{\longrightarrow} A \otimes A & A_{1} \otimes A_{2} \stackrel{\pi_{i}}{\longrightarrow} A_{i} \\
\text { Cf. } \quad A \vdash A \wedge A & A_{1} \wedge A_{2} \vdash A_{i} .
\end{array}
$$

Hence there is a direct connection to "resource-sensitive" logics such as linear $\operatorname{logic}[14]$.

A basic example, familiar to Computer Scientists, is given by Rel, the category with sets as objects and relations as arrows. Here the usual cartesian product of sets gives a tensor product, but not the categorical product. In particular, although we can define diagonals and projections, they are not natural. For diagonals, this means that the diagram

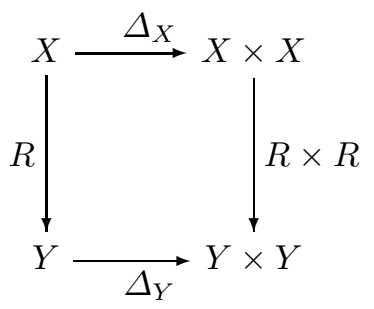

does not commute in general, where $\Delta_{X}=\{(x,(x, x)) \mid x \in X\}$ is the usual diagonal, and $R \subseteq X \times Y$ can be any relation.

A fundamental example for Quantum Mechanics and Quantum Information is FdHilb, the category of finite-dimensional complex Hilbert spaces and linear maps, with the standard concrete tensor product of linear algebra [4].

\section{Petri Nets and Monoidal Categories}

In the late 1980's there was a brief flowering of work relating Petri nets with monoidal categories and Linear logic $[29,13,21,20]$. This work does not seem 
to have had much lasting impact on the Petri net community, but the work by Ugo Montanari and José Meseguer in particular has been influential on wider developments in concurrency and graph rewriting, e.g. [22]. We shall briefly summarize their approach.

\subsection{The Meseguer and Montanari Approach}

Petri Nets are defined as

$$
N=\left(S^{\otimes}, T, \delta_{0}, \delta_{1}\right)
$$

where

$-S^{\otimes}$ is a free commutative monoid of states

$-T$ is the set of transitions

$-\delta_{0}, \delta_{1}: T \longrightarrow S^{\otimes}$ give the source and target of each transition.

A multiset of $S$-elements is just another way of thinking of a distribution of tokens.

Example

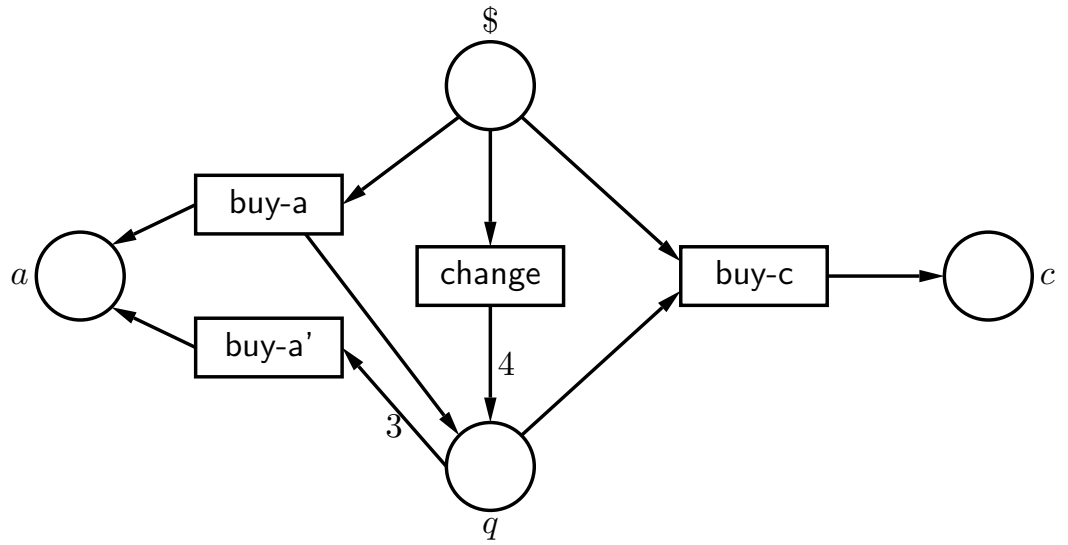

The transitions (axioms, arrows) are:

$$
\begin{aligned}
& \text { buy-c }: \$ \otimes q \longrightarrow c \text { buy-a }: \$ \longrightarrow a \otimes q \\
& \text { buy-a' }: q^{3} \longrightarrow a \quad \text { change }: \$ \longrightarrow q^{4}
\end{aligned}
$$

Petri Categories By closing up the transitions under sequential and parallel composition, a general notion of process is obtained:

- Given $\alpha: A \longrightarrow B$ and $\beta: B \longrightarrow C$, form $\alpha ; \beta: A \longrightarrow C$. Taking sequential composition to be associative, and adding idle transitions $1_{A}: A \longrightarrow A$ which are identities for sequential composition, this gives a category. 
- Given $\alpha: A_{1} \longrightarrow B_{1}$ and $\beta: A_{2} \longrightarrow B_{2}$, form the parallel composition $\alpha \otimes \beta: A_{1} \otimes A_{2} \longrightarrow B_{1} \otimes B_{2}$. If we assume the key (bi)functoriality axiom

$$
\left(\alpha_{1} \otimes \alpha_{2}\right) ;\left(\beta_{1} \otimes \beta_{2}\right)=\left(\alpha_{1} ; \beta_{1}\right) \otimes\left(\alpha_{2} ; \beta_{2}\right)
$$

this gives a symmetric monoidal category.

Meseguer and Montanari identify the particular kind of symmetric monoidal categories which arise in this way - the Petri categories. They show that the Best-Devillers theory of sequential and concurrent behaviours [8] can be recaptured in a systematic way in this framework.

\section{Processes in Monoidal Categories: A General Perspective}

We have seen that Petri Nets can be seen as particular examples of symmetric monoidal categories. But why not turn this around? Why not see any symmetric monoidal category as a setting for describing computational processes in a resource sensitive way, closed under sequential and parallel composition?

There is a natural objection to this, that we would then lose the additional concrete, combinatorial structure of Petri nets, and the corresponding graphical formalism, which is so much a part of how they are used.

But this objection does not really hold water! Monoidal categories, quite generally, admit a beautiful graphical calculus or diagrammatic notation which makes equational proofs perspicuous, and is sound and complete for equational reasoning in monoidal categories [15]. It also supports links with Logic (e.g. Proof Nets) and with Geometry (Knots, Braids, Temperley-Lieb algebra etc.) [1, $3]$.

\subsection{Outline of the Graphical Calculus}

In the graphical calculus we depict processes by boxes, and we label the inputs and outputs of these boxes by types which indicate the kind of system on which these processes act:

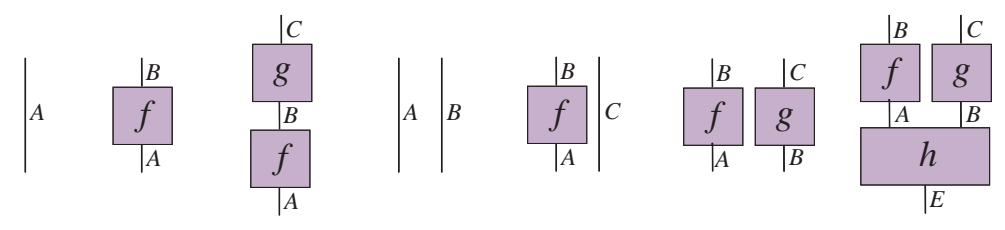

Algebraically, these correspond to:

$$
1_{A}: A \rightarrow A, \quad f: A \rightarrow B, \quad g \circ f, \quad 1_{A} \otimes 1_{B}, \quad f \otimes 1_{C}, \quad f \otimes g, \quad(f \otimes g) \circ h
$$

respectively. (The convention in these diagrams is that the 'upward' vertical direction represents progress of time.) 
Kets, Bras and Scalars: A special role is played by boxes with either no input or no output, i.e. arrows of the form $I \longrightarrow A$ or $A \longrightarrow I$ respectively, where $I$ is the unit of the tensor. In the setting of FdHilb and Quantum Mechanics, they correspond to states and costates respectively (cf. Dirac's kets and bras [12]), which we depict by triangles. Scalars then arise naturally by composing these elements (cf. inner-product or Dirac's bra-ket):

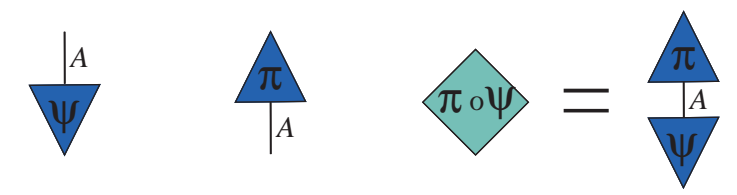

Formally, scalars are arrows of the form $I \longrightarrow I$. In the physical context, they provide numbers ("probability amplitudes" etc.). For example, in FdHilb, the tensor unit is $\mathbb{C}$, the complex numbers, and a linear map $s: \mathbb{C} \longrightarrow \mathbb{C}$ is determined by a single number, $s(1)$. In Rel, the scalars are the boolean semiring $\{0,1\}$.

This graphical notation can be seen as a substantial two-dimensional generalization of Dirac notation [12]:

$$
\langle\phi|\quad| \psi\rangle \quad\langle\phi \mid \psi\rangle
$$

Note how the geometry of the plane absorbs functoriality and naturality conditions, e.g.:

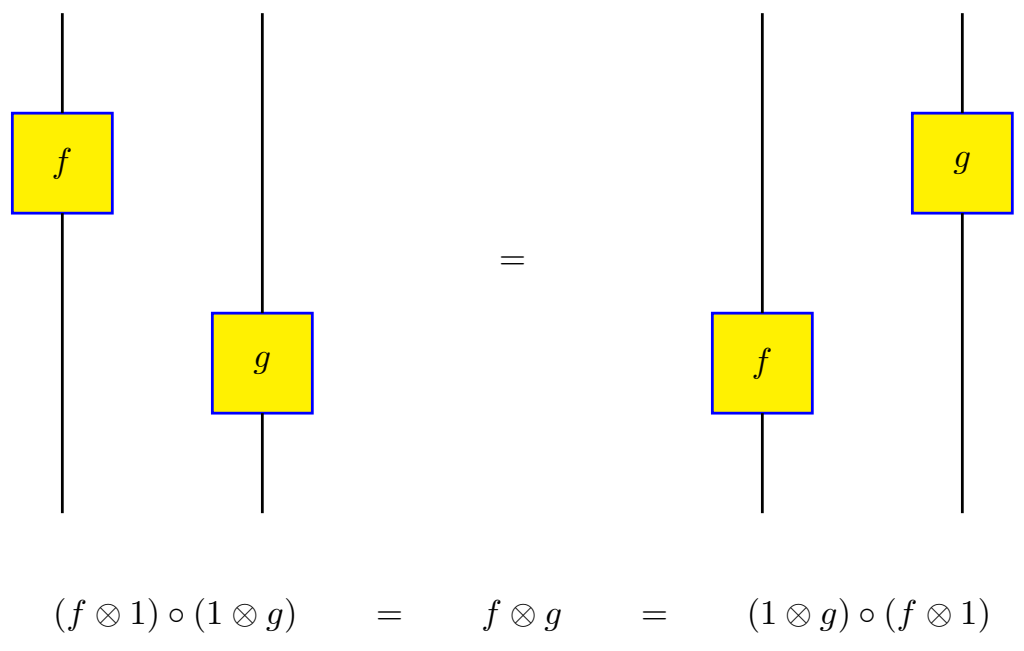

\section{Deficits and Cancellation}

We shall now consider an extension of the Meseguer-Montanari approach, due to Marti-Oliet and Meseguer [20]. For initial motivation, consider the following example: 
Example

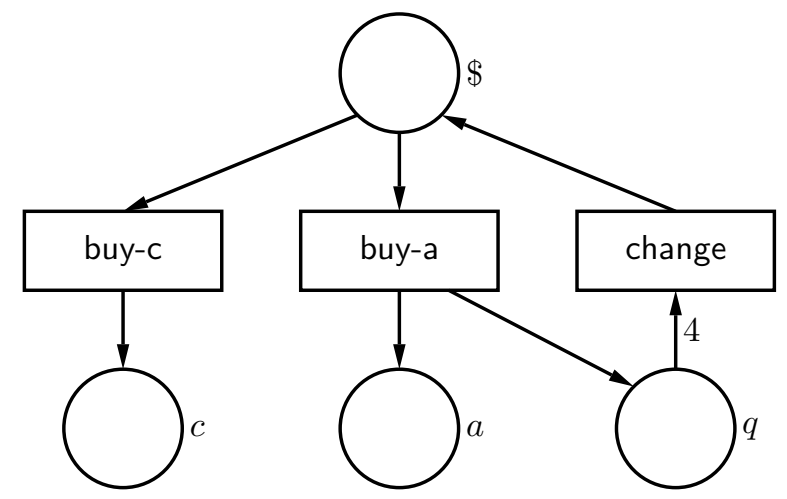

Transitions:

$$
\text { buy-c : } \$ \longrightarrow c \quad \text { buy-a }: \$ \longrightarrow a \otimes q \quad \text { change }: q^{4} \longrightarrow \$
$$

Note that there is no way of getting an apple if we start with three quarters!

\subsection{The Financial Game}

Marti-Oliet and Meseguer introduce the following modification of the usual token game, motivated by the aim of extending the connection between Petri Nets and monoidal structures to the whole of Multiplicative Linear Logic [14].

- Negative or dual tokens $a^{*}$ etc. are introduced. We can use these to represent situations involving deficits as well as the usual presence of resources.

- As well as the usual firing rules, we now have the opportunity to "borrow" resources, creating both the resource, and the corresponding deficit.

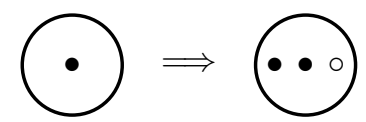

- Conversely, given a resource and a corresponding deficit, we can cancel them, removing both:

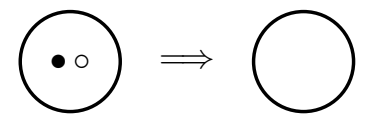

Deficits and Cancellation The basic transitions we need are:

$$
I \longrightarrow a^{*} \otimes a \quad a \otimes a^{*} \longrightarrow I
$$

where $I$ is the unit of the monoidal structure, creating and cancelling a deficit. 
We can now produce a computation in the above example to get an apple from three quarters:

$$
q^{3} \longrightarrow q^{3} \otimes I \longrightarrow q^{3} \otimes q^{*} \otimes q \longrightarrow \$ \otimes q^{*} \longrightarrow a \otimes q \otimes q^{*} \longrightarrow a
$$

This is a well known idea in Category theory: it takes us from symmetric monoidal to compact closed categories [16]. How does this look in our graphical calculus for monoidal categories?

Cups and Caps

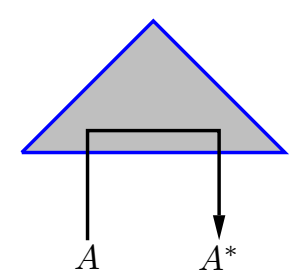

$\epsilon_{A}: A \otimes A^{*} \longrightarrow I$

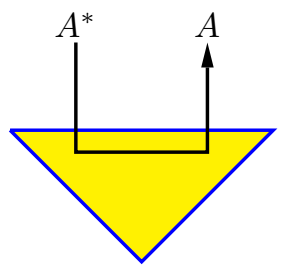

$\eta_{A}: I \longrightarrow A^{*} \otimes A$.

Caps $=$ Cancellations; Cups $=$ Deficits

\subsection{Graphical Calculus for Information Flow}

Compact Closure : The basic algebraic laws for units and counits.

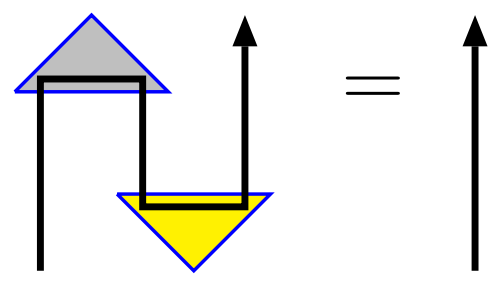

$\left(\epsilon_{A} \otimes 1_{A}\right) \circ\left(1_{A} \otimes \eta_{A}\right)=1_{A}$

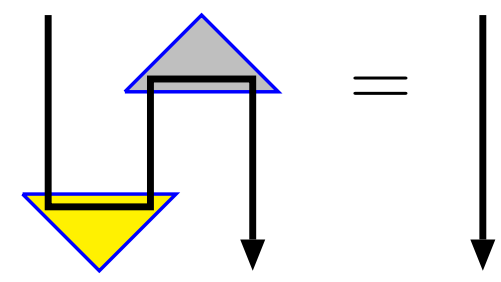

$\left(1_{A^{*}} \otimes \epsilon_{A}\right) \circ\left(\eta_{A} \otimes 1_{A^{*}}\right)=1_{A^{*}}$

In terms of deficits and cancellations:

$$
\odot \Rightarrow \odot=\odot=\odot
$$


Names and Conames in the Graphical Calculus The units and counits are powerful; they allow us to define a closed structure on the category. In particular, we can form the name $\ulcorner f\urcorner$ of any arrow $f: A \rightarrow B$, as a special case of $\lambda$-abstraction, and dually the coname $\llcorner f\lrcorner$ :

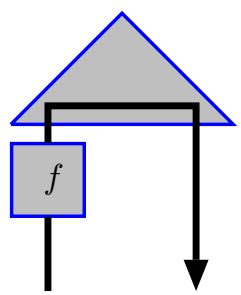

$\llcorner f\lrcorner: A \otimes B^{*} \rightarrow I$

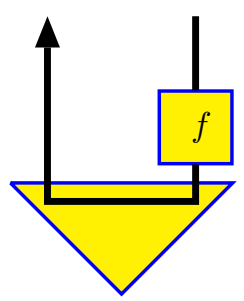

$\ulcorner f\urcorner: I \rightarrow A^{*} \otimes B$

This is the general form of Map-State duality:

$$
\mathcal{C}\left(A \otimes B^{*}, I\right) \simeq \mathcal{C}(A, B) \simeq \mathcal{C}\left(I, A^{*} \otimes B\right) .
$$

\section{Monoidal Categories and Physics}

Having related physics to Petri nets, and Petri nets to monoidal categories, we shall now show how to close the circle by relating (quantum) physics to monoidal categories, following [4]. Moreover, a key role will be played by the compact closed structure which was described in the previous section, as the abstract form of the "negative flows" of deficits and cancellations, introduced by MartiOliet and Meseguer to correspond to the logical structure of linear negation and implication. Here the same structure arises with a physical motivation, and plays a crucial rôle in explicating the information flows arising from quantum entanglement.

\subsection{Bits and Qubits}

\section{Classical Bits:}

- have two values 0,1

- are freely readable and duplicable

- admit arbitrary data transformations

\section{Qubits:}

- have a 'sphere' of values spanned by $|0\rangle,|1\rangle$ 

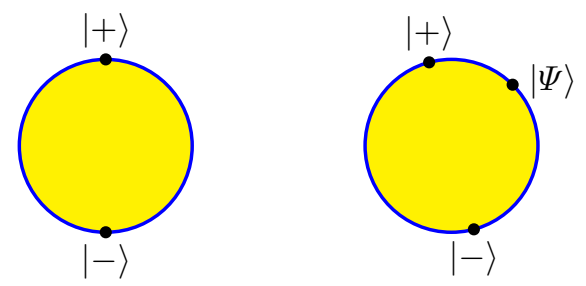

- measurements of qubits

- have two outcomes $|-\rangle,|+\rangle$

- change the value $|\psi\rangle$

- admit unitary transformations, i.e. antipodes and angles are preserved.

Formally, a qubit is a vector ${ }^{1}$ in the two-dimensional complex Hilbert space $\mathbb{C}^{2}$. This space allows for two degrees of freedom when we measure the qubit in a given basis; we get one of two possible answers, conventionally ' 0 ' or ' 1 ' in the standard basis. Which of these answers we get is in general uncertain; the state of the qubit tells us only the probability with which we will get each of the two possible answers. Moreover, measurement has an effect on the system being measured; it "collapses" to the basis state corresponding to the outcome of the measurement.

\subsection{Quantum Entanglement}

We consider for illustration two standard examples of two-qubit entangled states, the Bell state:

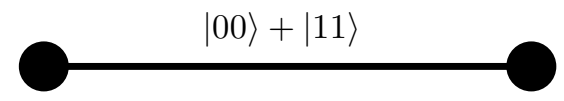

and the EPR state:

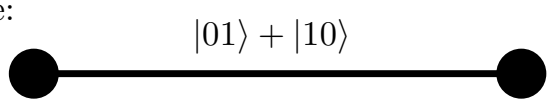

In quantum mechanics, compound systems are represented by the tensor product of Hilbert spaces: $\mathcal{H}_{1} \otimes \mathcal{H}_{2}$. A typical element of the tensor product has the form:

$$
\sum_{i} \lambda_{i} \cdot \phi_{i} \otimes \psi_{i}
$$

where $\phi_{i}, \psi_{i}$ range over basis vectors, and the coefficients $\lambda_{i}$ are complex numbers. Superposition encodes correlation: in the Bell state, the off-diagonal elements have zero coefficients. This gives rise to Einstein's "spooky action at a distance". Even if the particles are spatially separated, measuring one has an effect on the state of the other. In the Bell state, for example, when we measure one of the two qubits we may get either 0 or 1 , but once this result has been obtained, it is certain that the result of measuring the other qubit will be the same.

\footnotetext{
${ }^{1}$ Really by a one-dimensional subspace, or ray.
} 
This leads to Bell's famous theorem [6]: QM is essentially non-local, in the sense that the correlations it predicts exceed those of any "local realistic theory".

From 'paradox' to 'feature': Teleportation

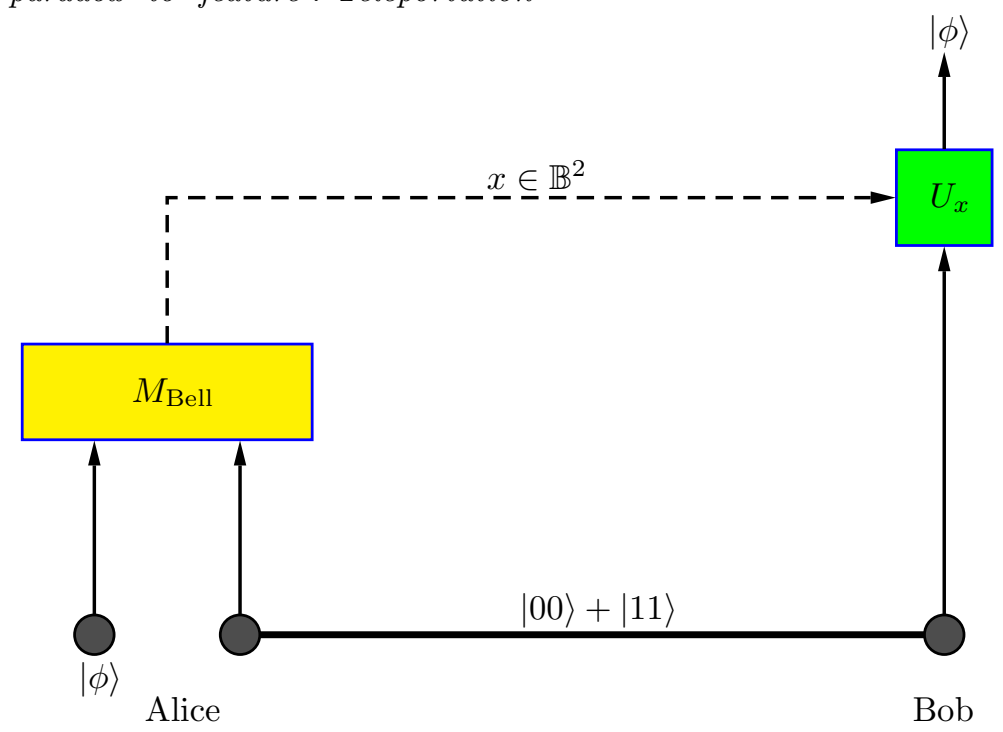

In the teleportation protocol [7], Alice sends an unknown qubit $\phi$ to Bob, using a shared Bell pair as a "quantum channel". By performing a measurement in the Bell basis on $\phi$ and her half of the entangled pair, a collapse is induced on Bob's qubit. Once the result $x$ of Alice's measurement is transmitted by classical communication to Bob (there are four possible measurement outcomes, hence this requires two classical bits), Bob can perform a corresponding unitary correction $U_{x}$ on his qubit, after which it will be in the state $\phi$.

\subsection{Categorical Quantum Mechanics and Diagrammatics}

We now outline the categorical approach to quantum mechanics developed in $[4,5]$. The same graphical calculus and underlying algebraic structure which we have seen in the previous section has been applied to quantum information and computation, yielding an incisive analysis of quantum information flow, and powerful and illuminating methods for reasoning about quantum informatic processes and protocols [4].

Bell States and Costates: The cups and caps we have already seen in the guise of deficit and cancellation operations, now take on the rôle of Bell states and costates (or preparation and test of Bell states), the fundamental building blocks of quantum entanglement. (Mathematically, they arise as the transpose and co-transpose of the identity, which exist in any finite-dimensional Hilbert space by "map-state duality"). 

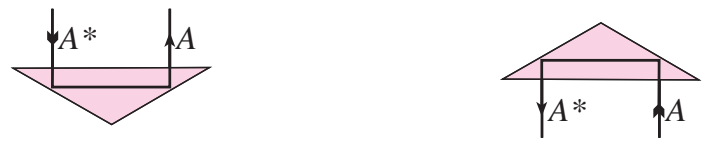

The formation of names and conames of arrows (i.e. map-state and mapcostate duality) is conveniently depicted thus:

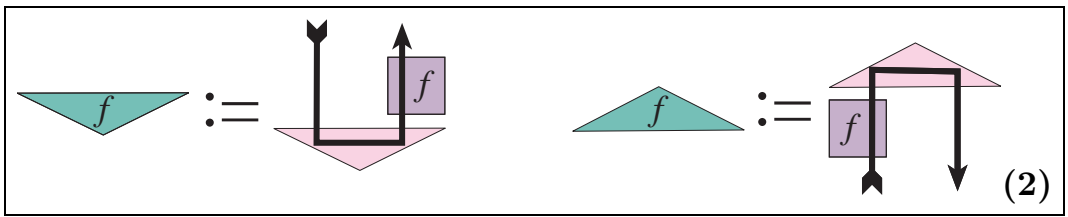

The key lemma in exposing the quantum information flow in (bipartite) entangled quantum systems can be formulated diagrammatically as follows:

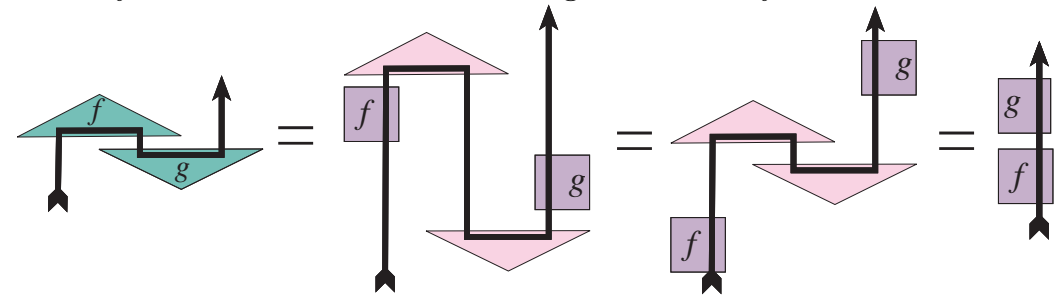

Note in particular the interesting phenomenon of "apparent reversal of the causal order" . While on the left, physically, we first prepare the state labeled $g$ and then apply the costate labeled $f$, the global effect is as if we first applied $f$ itself first, and only then $g$. This corresponds to the apparent reversal of flow of computations in the token game on Petri nets achieved with deficits and cancellations.

Derivation of quantum teleportation. This is the most basic application of compositionality in action. We can read off the basic quantum mechanical potential for teleportation immediately from the geometry of Bell states and costates:

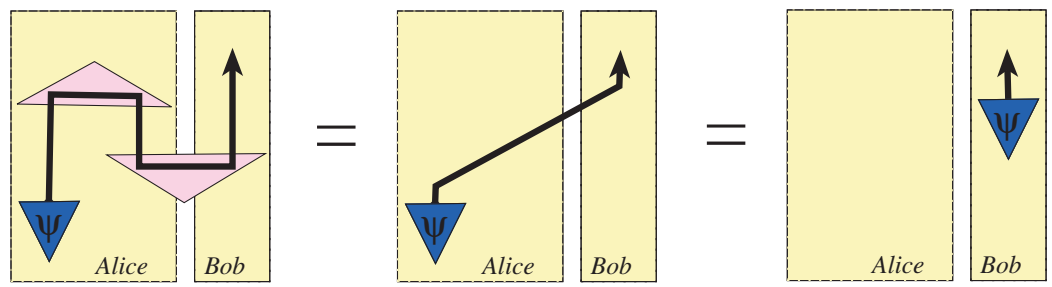

The Bell state forming the shared channel between Alice and Bob appears as the downwards triangle in the diagram; the Bell costate forming one of the possible measurement branches is the upwards triangle. The information flow of the input qubit from Alice to Bob is then immediately evident from the diagrammatics.

This is not quite the whole story, because of the non-deterministic nature of measurements. But in fact, allowing for this shows the underlying design 
principle for the teleporation protocol. Namely, we find a measurement basis such that each possible branch $i$ through the measurement is labelled, under map-state duality, with a unitary map $f_{i}$. The corresponding correction is then just the inverse map $f_{i}^{-1}$. Using our lemma, the full description of teleportation becomes:

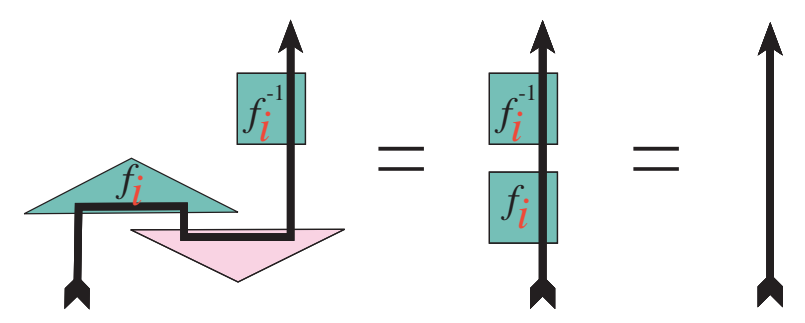

\section{Conclusions}

We have described a striking nexus of ideas arising from several different sources. Conceptually, the most interesting feature has been the need for "negative information flow", which has arisen from several sources:

- In logic, with the need to account for negative polarities, as created by connectives such as (linear) negation or implication, leading to Marti-Oliet and Meseguer's proposal of deficits and cancellations as a computational correlate in the setting of Petri net dynamics.

- Physically, e.g. in the desciption of quantum teleportation, these negative flows run counter to the normal flow of time and causality, and form part of the enigma of quantum mechanics.

- Mathematically, we are led to dualities and closed structure.

- Geometrically, we go in the direction of loops, tangles and knots.

Standard Petri net theory, with its careful enforcement of local causality and information flow, explicitly influenced by relativity theory, does not suffice to capture the non-local features of quantum mechanics, which are exploited in quantum information. Perhaps it is not too fanciful to see here in microcosm some of the foundational obstacles to formulating an adequate theory of quantum gravity!

The further elaboration and deepening of this nexus of ideas offers many interesting challenges. In particular, a full analysis of distributed quantum computation, e.g. quantum security protocols, which should take into account both the quantum and the classical ingredients of the protocols, and also reflect enough of the spatio-temporal structure to capture the salient distributed features, will require a deeper understanding of the ingredients we have assembled here, and of how they may be related and combined. 


\section{References}

1. Abramsky, S. (2005) Abstract Scalars, Loops, and Free Traced and Strongly Compact Closed Categories. In Proceedings of CALCO 2005, Springer LNCS Vol. 3629, 1-31, 2005.

2. S. Abramsky. What are the fundamental structures of concurrency? We still don't know!, in Proceedings of the Workshop "Essays on Algebraic Process Calculi" (APC 25). Electronic Notes in Theoretical Computer Science, Volume 162:37-41, 2006 .

3. S. Abramsky. Temperley-Lieb algebra: from knot theory to logic and computation via quantum mechanics. In Mathematics of Quantum Computation and Quantum Technology, G. Chen, L. Kauffman and S. Lomonaco, eds., Taylor and Francis, pages 515-558, 2007.

4. Abramsky, S. and B. Coecke. A categorical semantics of quantum protocols. In Proceedings of the 19th Annual IEEE Symposium on Logic in Computer Science, 415-425, 2004. arXiv: quant-ph/0402130.

5. Abramsky, S. and B. Coecke, Abstract physical traces. Theory and Applications of Categories 14 (2005), 111-124.

6. J. S. Bell. On the Problem of Hidden Variables in Quantum Mechanics. Reviews of Modern Physics, 1966

7. C. H. Bennet, G. Brassard, C. Crépeau, R. Jozsa, A. Peres, and W. K. Wooters. (1993). Teleporting an unknown quantum state via dual classical and EinsteinPodolsky-Rosen channels. Physical Review Letters, 70:1895-1899.

8. E Best and R Devillers. Sequential and concurrent behaviour in Petri net theory. Theoretical Computer Science, 1987.

9. L. Bombelli, J. Lee, D. Meyer and R.D. Sorkin, Spacetime as a causal set, Phys. Rev. Lett. 59: 521-524 (1987).

10. L. Cardelli and A. D. Gordon. Mobile Ambients. FoSSaCS 1998: 140-155. Springer LNCS Volume 1378, 1998.

11. R. Carnap. Introduction to Symbolic Logic with Applications. Dover Books, 1958.

12. P. A. M. Dirac. (1947). The Principles of Quantum Mechanics (third edition). Oxford University Press.

13. V. Gehlot and C. Gunter, Normal process representatives, Proceedings LiCS 1990.

14. J.-Y. Girard, Linear Logic. Theoretical Computer Science 50(1):1-102, 1987.

15. A. Joyal and R. Street. (1991). The geometry of tensor calculus I. Advances in Mathematics, 88:55-112.

16. G. M. Kelly and M. L. Laplaza. (1980). Coherence for compact closed categories. Journal of Pure and Applied Algebra, 19:193-213.

17. R. Landauer, Information is physical, Physics Today, 44:May(1991), 23-29.

18. S. Mac Lane. Categories for the Working Mathematician, 2nd Edition, Springer 1998.

19. K. Martin and P. Panangaden. A Domain of spacetime intervals for General Relativity. Communications of Mathematical Physics, November 2006.

20. N. Marti-Oliet and J. Meseguer, From Petri Nets To Linear Logic Through Categories, IJFCS 2(4):297-399, 1991.

21. J. Meseguer and U. Montanari, Petri Nets Are Monoids, Information and Computation 88:105-155, 1990.

22. R. Milner. Calculi for Interaction. Acta Informatica 33, 1996.

23. R. Milner. Pure bigraphs: Structure and dynamics. Inf. Comput. 204(1): 60-122 (2006). 
24. C. A. Petri. Fundamentals of a Theory of Asynchronous Information Flow. IFIP Congress 1962: 386-390.

25. Petri, C.-A., State-Transition Structures in Physics and in Computation. International Journal of Theoretical Physics, 21(12), (1982) 979-993.

26. C. A. Petri. Nets, Time and Space. Theor. Comput. Sci. 153(1-2): 3-48 (1996).

27. L. Smolin. Three Roads to Quantum Gravity. Phoenix 2000.

28. R.D. Sorkin, A Specimen of Theory Construction from Quantum Gravity, in J. Leplin (ed.), The Creation of Ideas in Physics: Studies for a Methodology of Theory Construction (Proceedings of the Thirteenth Annual Symposium in Philosophy, held Greensboro, North Carolina, March, 1989) pp. 167-179, Kluwer Academic Publishers, Dordrecht, 1995.

29. G. Winskel, Petri nets, algebras, morphisms and compositionality. Information and Computation 72:197-238, 1987. 